\title{
Pediatric cancer deaths: curative or palliative?
}

\begin{abstract}
Aims: The aims of this study were to review the deaths of Malaysian pediatric oncology patients in order to determine the major causes and the proportion of patients who received palliative care. Subjects and methods: This was a retrospective review from 2001-2007 of deaths at the Pediatric Institute, General Hospital Kuala Lumpur. Clinical data such as age, gender, disease, cause, and place of death were collected. The patients were divided into two groups: those who received palliative care and those who received curative treatment. Results: Two hundred forty-seven patients were included. There were 148 males and 99 females. The age ranged from 2 months to 22 years (median, 4 years). One hundred thirty cases $(53 \%)$ were still in the curative phase of treatment at the time of death. The most common cause of death was septicemia (62\%), followed by hemorrhage (18\%) and underlying cancer (14\%). One hundred seventeen cases $(47 \%)$ were in the palliative phase at the time of death. All palliative care deaths occurred in the oncology ward with one exception. Conclusion: Nearly half of all inpatient pediatric cancer deaths were palliative in nature. Septicemia and hemorrhage were the major causes of death in the others.
\end{abstract}

